\title{
Metformin alleviates $\beta$-glycerophosphate-induced calcification of vascular smooth muscle cells via AMPK/mTOR-activated autophagy
}

\author{
XIAOBO QIU ${ }^{1,3}$, QING XU², TIANHUA XU ${ }^{1}$, PENGZHI WAN ${ }^{1}$, ZITONG SHENG ${ }^{1}$, YIRAN HAN ${ }^{1}$ and LI YAO ${ }^{1}$ \\ ${ }^{1}$ Department of Nephrology, The First Hospital of China Medical University, Shenyang, Liaoning 110000; \\ ${ }^{2}$ Department of Orthopaedics, Shengjing Hospital of China Medical University, Shenyang, Liaoning 110004, P.R. China
}

Received September 17, 2019; Accepted September 11, 2020

DOI: $10.3892 /$ etm.2020.9490

\begin{abstract}
The aim of the present study was to investigate the effect of metformin on $\beta$-glycerophosphate-induced calcification of vascular smooth muscle cells (VSMCs) and the possible mechanisms underlying this. Using an established VSMC calcification model, VSMCs were first treated with $\beta$-glycerophosphate, before metformin, 3-methyladenine and compound $\mathrm{C}$ were added to the cell cultures in different combinations. Calcium deposition in the cells was examined by Alizarin Red S staining and using the O-cresolphthalein complexone method. To assess the occurrence of autophagy, autophagosomes inside the cells were studied using a transmission electron microscope and green fluorescent microtubule-associated protein 1 light chain 3 (LC3) puncta were examined using a fluorescent microscope. Additionally, protein expression levels of $\alpha$-smooth muscle actin ( $\alpha$-SMA), runt-related transcription factor 2 (RUNX2), LC3II/I, beclin 1 and $5^{\prime}$ adenosine monophosphate-activated protein kinase (AMPK)/mammalian target of rapamycin (mTOR) pathway-associated proteins were determined by western blot analysis. Metformin increased the number of autophagosomes, green fluorescent LC3 puncta and the levels of LC3II/I, beclin 1, $\alpha$-SMA and phosphorylated (p)-AMPK in the VSMCs that were treated with $\beta$-glycerophosphate when compared to controls; whereas, calcium deposition and the expression levels of RUNX2 and p-mTOR were found to be decreased. Treating the VSMCs with 3-methyladenine or
\end{abstract}

Correspondence to: Professor Li Yao, Department of Nephrology, The First Hospital of China Medical University, 155 Nanjing North Street, Shenyang, Liaoning 110000, P.R. China

E-mail: liyao_cmu@163.com

Present address: ${ }^{3}$ Tianjin Children's Hospital, Tianjin 300074, P.R. China

Key words: metformin, $\beta$-glycerophosphate, vascular smooth muscle cell, autophagy, vascular calcification, chronic kidney disease compound $\mathrm{C}$ reversed the effects of metformin. The results of the present study suggested that metformin may alleviate $\beta$-glycerophosphate-induced calcification of VSMCs, which may be attributed to the activation of AMPK/mTOR signaling pathway-dependent autophagy.

\section{Introduction}

Chronic kidney disease (CKD) is a major health problem faced by individuals worldwide (1). Atherosclerosis and vascular calcification are highly prevalent in patients with CKD and are known to be major risk factors for all-cause and cardiovascular mortalities in these patients (2). Multiple mechanisms are thought to be associated with vascular calcification, including hyperphosphatemia, oxidative stress, inflammation and apoptosis; however, none of these mechanisms is fully understood (3). Clinical and epidemiological studies have shown that hyperphosphatemia is the most important risk factor associated with the initiation of vascular calcification in patients with CKD, leading to the hypothesis that maintaining serum phosphorus at appropriate levels may significantly alleviate vascular calcification and improve clinical outcomes (4). Moreover, osteochondrocytic differentiation of vascular smooth muscle cells (VSMCs) under high phosphate conditions is considered to be a pivotal pathological process in the initiation of vascular calcification (5).

Studies have indicated that autophagy plays an important role in the function of VSMCs and in the development of vascular diseases, suggesting that it may be a potential target in the prevention of vascular calcification (6-8). Autophagy induced by high phosphate conditions has been demonstrated to counteract the phosphate-induced calcification of VSMCs (9). High phosphorus promotes calcification of VSMCs and activates autophagy, which maintains VSMC stability and allows VSMCs to adapt to unfavorable conditions (9). First-line drugs used for treating hyperglycemia, such as metformin, also exhibit protective effects on the cardiovascular system; however, the underlying mechanisms are not fully understood. It has previously been reported that metformin can activate 5' adenosine monophosphate-activated protein kinase (AMPK) (10) and the AMPK/mammalian target of rapamycin (mTOR) signaling pathway has been shown to regulate autophagy, both directly 
and indirectly (11). AMPK could initiate autophagy either by directly phosphorylating serine/threonine-protein kinase ULK1 protein (12), or indirectly by deactivating mTORC1 (13). The aim of the present study was to investigate the function of metformin in the alleviation of $\beta$-glycerophosphate-induced calcification of VSMCs and the role of AMPK/mTOR-activated autophagy in this pathological condition.

\section{Materials and methods}

Cell culture. The rat thoracic aorta VSMC line A7r5 was purchased from The Cell Bank of Type Culture Collection of the Chinese Academy of Sciences the Shanghai Institutes for Biological Sciences. The cells were inoculated in $35-\mathrm{mm}$ dishes at a density of $2 \times 10^{4}$ cells and were cultured in high-glucose Dulbecco's modified Eagle's medium (HDMEM) containing 10\% fetal bovine serum (FBS) (both from Hyclone; Cytiva) unless otherwise indicated. The cell cultures were incubated at $37^{\circ} \mathrm{C}$ in a humidified thermostatic incubator supplied with $5 \% \mathrm{CO}_{2}$. Medium was refreshed every 2-3 days. To induce calcification, the VSMCs were cultured in $1 \%$ FBS-HDMEM containing $10 \mathrm{mmol} / \mathrm{l} \beta$-glycerophosphate $(\beta$-GP; Sigma-Aldrich; Merck KGaA) for 14 days $(9,14)$. To determine the effect of metformin on the calcification of VSMCs and the underlying mechanisms involved, VSMCs were incubated in $1 \%$ FBS-HDMEM containing $10 \mathrm{mmol} / 1 \beta$-GP and $500 \mu \mathrm{mol} / \mathrm{metformin}$ (Sigma-Aldrich; Merck KGaA), as described previously (15). To investigate the effect of autophagy on the calcification of VSMCs, the cells were pre-treated with the autophagy inhibitor, 3-methyladenine (3-MA; Sigma-Aldrich; Merck KGaA) at $5 \mathrm{mmol} / \mathrm{l}$ for $30 \mathrm{~min}$ (9). To investigate the involvement of the AMPK/mTOR signaling pathway in the induction of autophagy by metformin, the VSMCs were pre-treated with the AMPK inhibitor compound C (Sigma-Aldrich; Merck KGaA) at $10 \mu \mathrm{mol} / 1$ for 30 min (16). VSMCs cultured in 1\% FBS-HDMEM were used as the control.

Alizarin Red S staining and quantification of cell calcium content. After treatment with $\beta$-GP for 14 days, VSMCs were fixed in $95 \%$ ethanol for $1 \mathrm{~h}$ at room temperature. The cells were stained with $1 \%$ Alizarin Red S solution (Sigma-Aldrich; Merck KGaA) for 30 min at $37^{\circ} \mathrm{C}$. Cells were then washed three times with PBS to remove non-specific staining. The positively-stained mineralized matrix exhibited a reddish/purple color upon direct visualization. Mineralized nodules were observed at $\mathrm{x} 40$ magnification under an inverted microscope (Nikon TMS; Nikon Corporation) with three fields for each group. In order to quantify their calcium content, VSMCs were incubated in triplicate for 14 days before subjecting them to decalcification with $0.6 \mathrm{~mol} / \mathrm{l}$ hydrochloric acid $(\mathrm{HCl})$ for $24 \mathrm{~h}$ at $37^{\circ} \mathrm{C}$. The calcium content in the $\mathrm{HCl}$ supernatants was determined for each sample using the O-cresolphthalein complexone method (17). Total protein from the treated cells was extracted using $1 \mathrm{M} \mathrm{NaOH} / 0.1 \%$ SDS and was quantified using the BCA method. The calcium content was normalized to the protein content and was expressed as $\mu \mathrm{g}$ calcium per mg protein.

Transmission electron microscopy (TEM). TEM was performed to assess the formation of autophagosomes in the
VSMCs. Briefly, after culture for $72 \mathrm{~h}$, VSMCs were pre-fixed with $2.5 \%$ glutaraldehyde for $2 \mathrm{~h}$ at room temperature and post-fixed with $1 \%$ osmium tetroxide for $2 \mathrm{~h}$ at $4^{\circ} \mathrm{C}$. After performing the dehydration process in an ethanol gradient (50\% for $15 \mathrm{~min}, 70 \%$ overnight, followed by 80 and $90 \%$ each for $15 \mathrm{~min}$, and $100 \%$ twice, each for $20 \mathrm{~min}$ ), the cells were incubated with propanone and embedded in SPI-Pon-812 resin (Structure Probes, Inc.). Ultrathin sections were prepared using an ultramicrotome EM UC7 (Leica Microsystems GmbH), and were then stained with uranyl acetate and lead nitrate. The stained sections were examined under the FEI Tecnai G2 Spirit transmission electron microscope (Thermo Fisher Scientific, Inc.). Three fields of each section were examined and photographed.

Immunofluorescence. Immunofluorescence analysis was performed to examine the expression of microtubule-associated protein 1 light chain 3 (LC3). Briefly, the cells were inoculated in 24-well plates at a density of $3 \times 10^{4}$ cells/well. After culture for $72 \mathrm{~h}$, VSMCs were fixed with $4 \%$ paraformaldehyde for $20 \mathrm{~min}$ at room temperature and were permeablized by incubation with $0.5 \%$ Triton X-100 for $5 \mathrm{~min}$ at room temperature. Thereafter, the cells were blocked in $100 \%$ goat serum blocking solution (Beyotime Institute of Biotechnology) for $2 \mathrm{~h}$ at room temperature, followed by incubation with anti-LC3 mAb (1:200; cat. no. 3868; Cell Signaling Technology, Inc.) overnight at $4^{\circ} \mathrm{C}$. The cells were then incubated with FITC-conjugated secondary antibody (1:150; cat. no. WLA032; Wanleibio Co., Ltd.) in the dark for $2 \mathrm{~h}$ at room temperature. Nuclei of the cells were stained with DAPI for $5 \mathrm{~min}$ at room temperature. The cells were visualized under a fluorescent microscope at x200 magnification. Three fields of each sample were examined and photographed.

Western blot analysis. Total protein from the VSMCs was extracted using RIPA lysis buffer containing protease and phosphatase inhibitors (all from Beyotime Institute of Biotechnology). The protein content in the extracts was quantified using the BCA method. Equal amounts of protein $(40 \mu \mathrm{g})$ were subjected to SDS-PAGE [6\% for LC3, 12\% for mTOR and phosphorylated (p)-mTOR, and $10 \%$ for all other proteins], followed by the transfer of proteins onto a PVDF membrane. The membranes were incubated successively with $5 \%$ non-fat milk for $1 \mathrm{~h}$ at room temperature, with appropriate primary antibodies overnight at $4^{\circ} \mathrm{C}$, and with horseradish peroxidase-conjugated secondary antibody $(1: 2,000$; cat. no. WLA023a; Wanleibio Co., Ltd.) for $1 \mathrm{~h}$ at room temperature. The following primary antibodies were used: Anti-LC3 (1:500; cat. no. 3868; Cell Signaling Technology, Inc.), anti-beclin 1 (1:500; cat. no. 3495; Cell Signaling Technology, Inc.), anti- $\alpha$-SMA (1:1,000; cat. no. 19245 ; Cell Signaling Technology, Inc.), anti-RUNX2 (1:1,000; cat. no. 12556; Cell Signaling Technology, Inc.), anti-AMPK (1:500; cat. no. 2532; Cell Signaling Technology, Inc.), anti-Thr172p-AMPK (1:500; cat. no. 2535; Cell Signaling Technology, Inc.), anti-mTOR (1:1,000; cat. no. ab32028; Abcam), anti-ser2448p-mTOR (1:1,000; cat. no. ab109268; Abcam), anti-GAPDH (1:1,000; cat. no. ab181602; Abcam) and anti- $\beta$-actin (1:1,000; cat. no. ab179467; Abcam). The membranes were developed using the ECL (Wanleibio Co., 
A

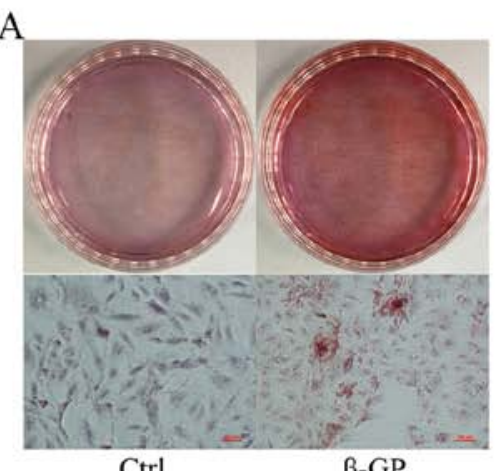

Ctrl

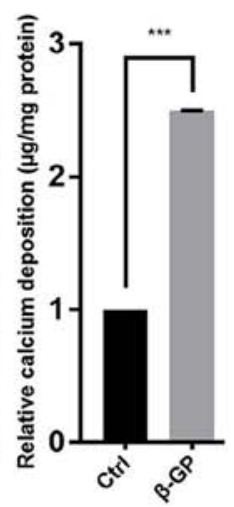

B

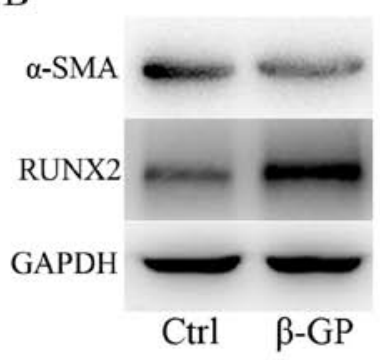

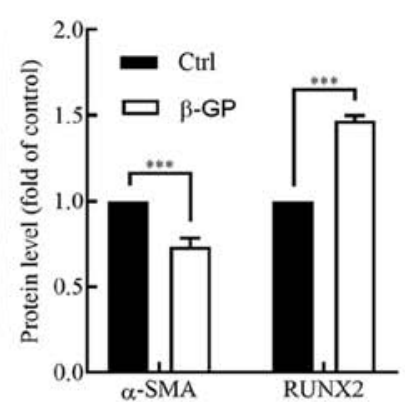

C

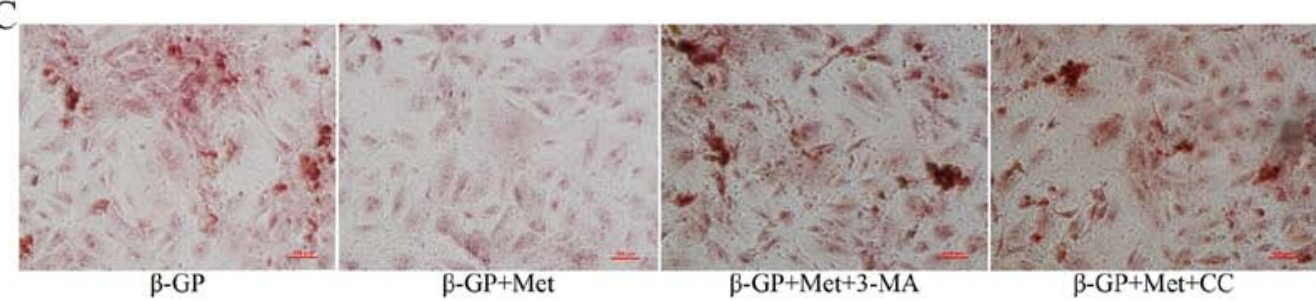

D
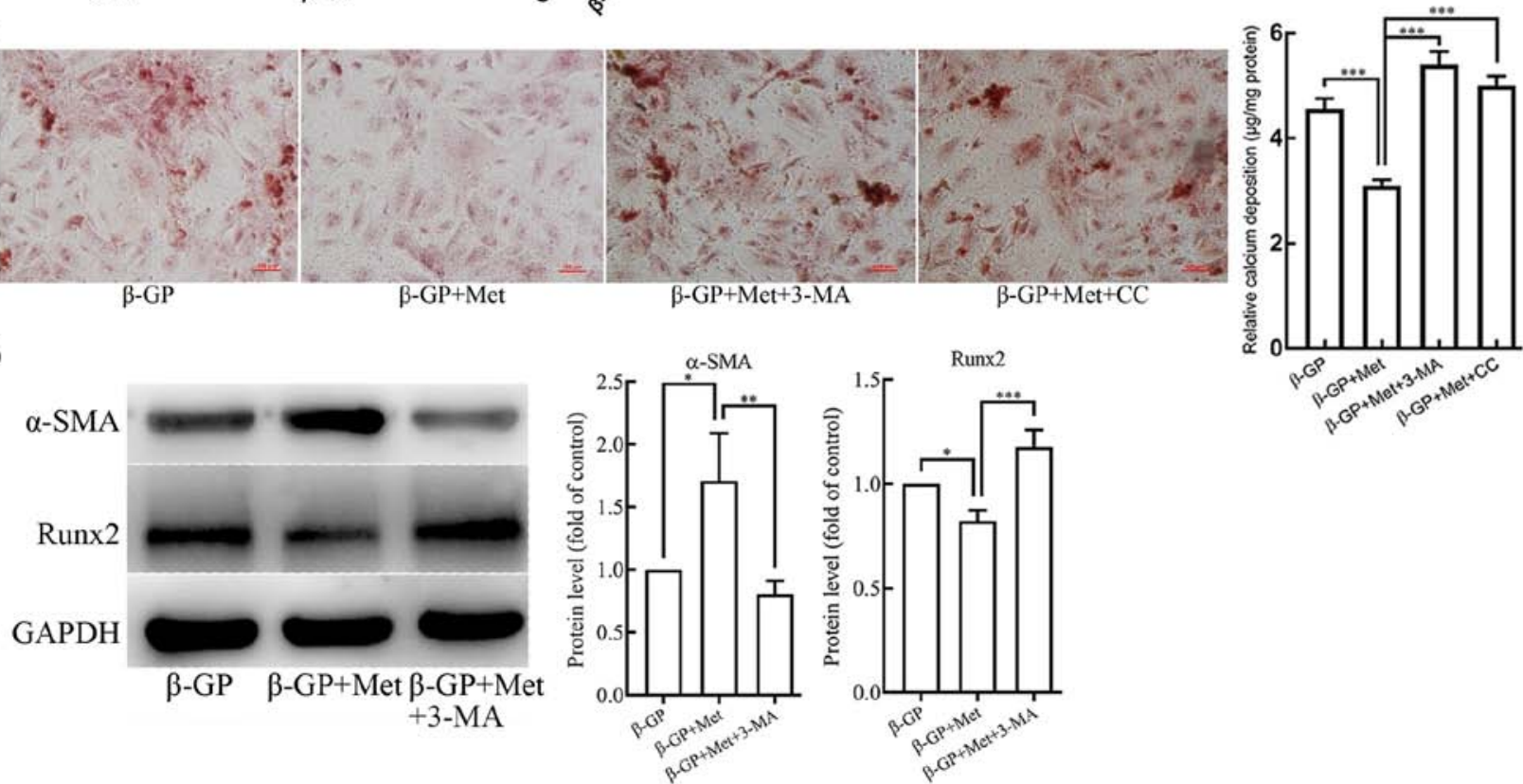

Figure 1. The effects of $\beta$-GP, Met, 3-MA and CC on VSMC calcification. VSMCs were treated with $\beta$-GP (10 mmol/1) to induce calcification and with metformin $(500 \mu \mathrm{mol} / \mathrm{l})$ to examine its effect on calcification. 3-MA (5 mmol/1) and CC (10 $\mu$ mol/1) were added to the cells to investigate the effects of autophagy and the AMPK signaling pathway, respectively. (A) Alizarin red S staining and O-cresolphthalein complexone method calcium quantitation showing calcium deposition in VSMCs treated with vehicle or $\beta$-GP. (B) Western blots showing protein levels of $\alpha$-SMA and Runx 2 in VSMCs treated with vehicle or $\beta$-GP. (C) Alizarin red S staining and O-cresolphthalein complexone method calcium quantitation showing calcium deposition in VSMCs treated with $\beta$-GP, MET, 3-MA, or CC. (D) Western blots showing protein levels of $\alpha$-SMA and Runx2 in VSMCs treated with $\beta$-GP, Met, or 3-MA. Western bands of interest were normalized against GAPDH, and data are provided as relative density ratios compared with control group or $\beta$-GP group. Scale bars in Alizarin red $\mathrm{S}$ staining represent $100 \mu \mathrm{m}$. Representative images are shown. Data are presented as mean $\pm \mathrm{SEM}, \mathrm{n}=3$. ${ }^{*} \mathrm{P}<0.05,{ }^{* * *} \mathrm{P}<0.01,{ }^{* * *} \mathrm{P}<0.001$. $\beta$-GP, $\beta$-glycerophosphate; Met, metformin; 3-MA, 3-methyladenine; CC, compound C; VSMC, vascular smooth muscle cell; $\alpha$-SMA, $\alpha$-smooth muscle actin; RUNX2, runt-related transcription factor 2 .

Ltd.) detection system. All the bands obtained were analyzed using the ImageJ software (National Institutes of Health). Normalization of the protein bands was performed using GAPDH or $\beta$-actin levels. Data are presented as relative density ratio of the $\beta$-GP group compared with control group in initial experiments After finding that $\beta$-GP influenced protein expression levels compared to the control group, further experiments were performed to compare the effect of treatment with a combination of $\beta$-GP metformin, 3-MA and Compound $\mathrm{C}$ in comparison with $\beta$-GP alone.

Statistical analysis. Each experiment was performed in triplicate. Comparisons between two groups were performed using unpaired t-test. Comparisons between the values of three or more groups were performed using one-way ANOVA, followed by the Tukey's multiple comparison test. All the analyses in this study were performed using GraphPad Prism software (version 7.04; GraphPad Software, Inc.). The results of the experiments are presented as the mean \pm SEM and $\mathrm{P}<0.05$ was considered statistically significant.

\section{Results}

Metformin alleviates $\beta$-glycerophosphate-induced VSMC calcification. VSMCs were treated with $\beta$-GP $(10 \mathrm{mmol} / \mathrm{l})$ in order to induce calcification. Alizarin Red S staining and O-cresolphthalein complexone assay (Fig. 1A) revealed that $\beta$-GP enhanced calcium deposition in VSMCs when compared to that in the untreated control cells. In addition, the protein expression level of the smooth muscle cell marker $\alpha$-SMA was found to be reduced in $\beta$-GP treated cells compared with the untreated control, whereas the protein expression level of the osteogenic gene RUNX2 was increased, as shown in the western blotting analysis (Fig. 1B). To determine whether 

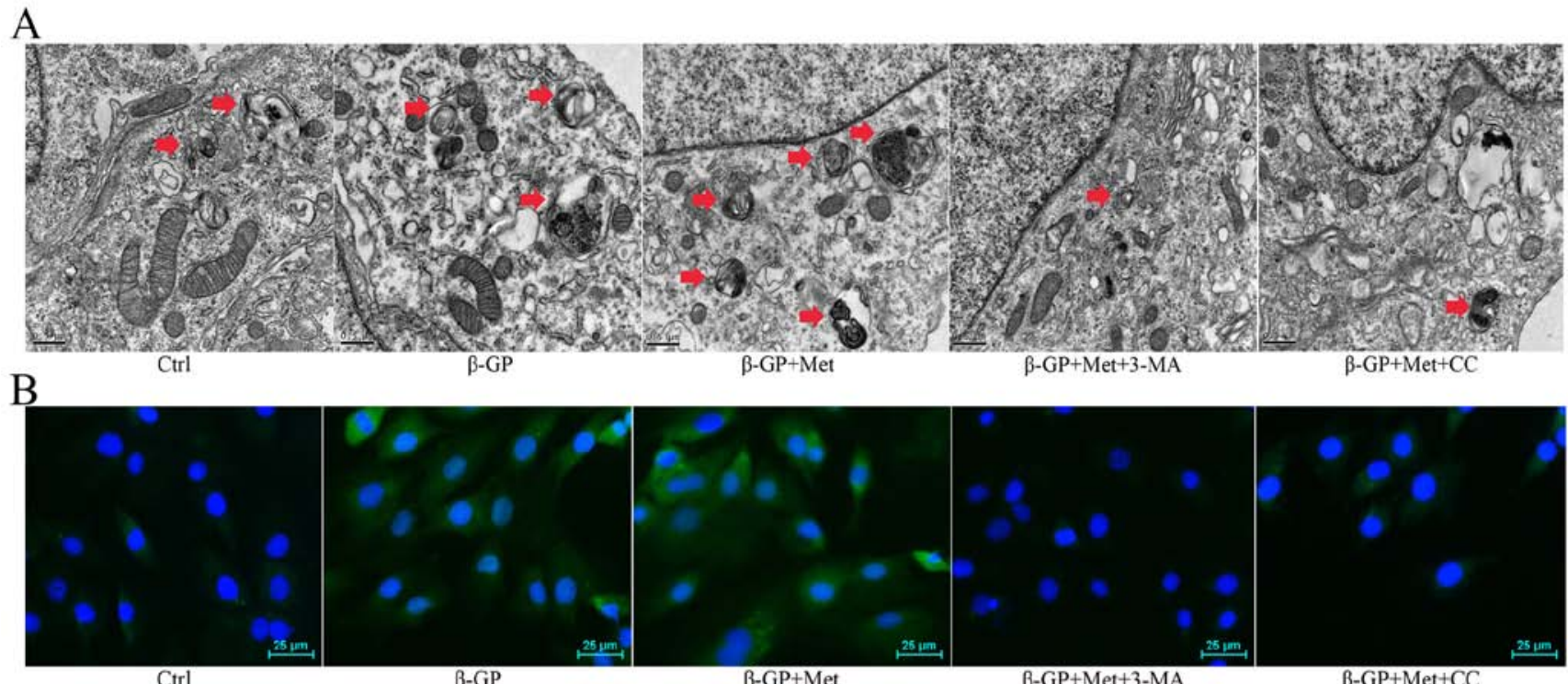

$\beta-G P$

$\beta-G P+M e$

$\beta-G P+M e t+3-M A$

$\beta-\mathrm{GP}+\mathrm{Met}+\mathrm{CC}$

Ctrl

C

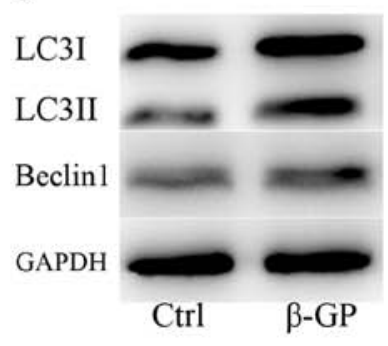

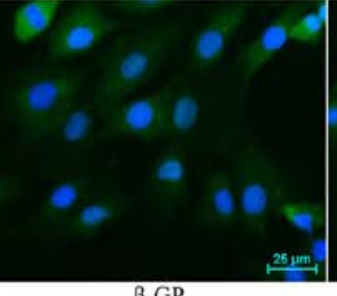

$\beta-G P$

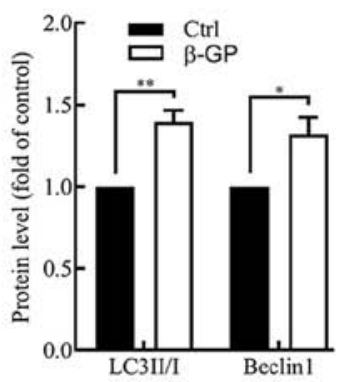

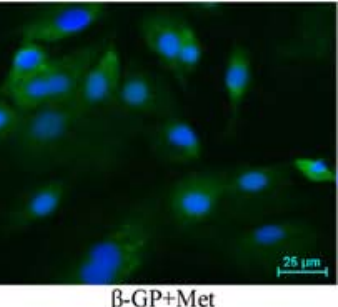

D

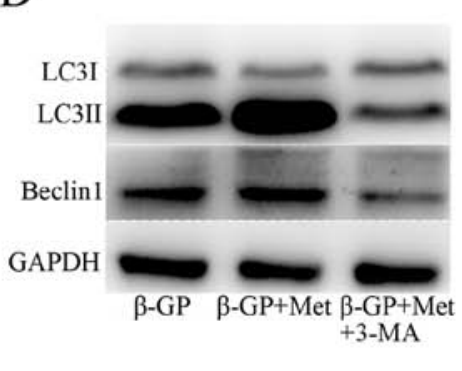

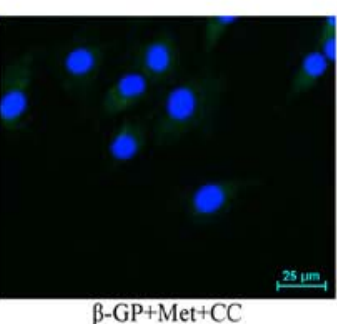

$\beta-\mathrm{GP}+\mathrm{Met}+\mathrm{CC}$
$\beta-G P+M e t+3-M A$

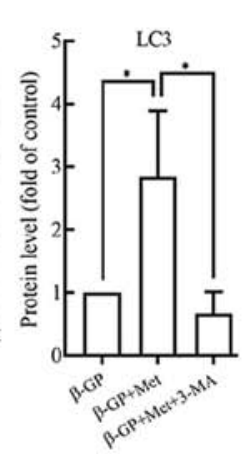

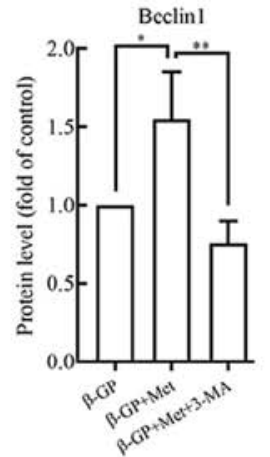

Figure 2. The effects of $\beta$-GP, Met, 3-MA and CC on autophagic activity in VSMCs. (A) TEM showing autophagosomes (red arrows) in VSMCs treated with $\beta$-GP, Met, 3-MA or CC. Scale bars represent $0.5 \mu \mathrm{m}$. (B) Immunofluorescence staining showing the expression of LC3 in VSMCs treated with $\beta$-GP, Met, 3 -MA, or CC. Scale bars represent $25 \mu \mathrm{m}$. Cells were treated with $1 \%$ FBS-HDMEM containing $\beta$-GP, $\beta$-GP + Met, $\beta$-GP + Met $+3-\mathrm{MA}, \beta-\mathrm{GP}+\mathrm{Met}+\mathrm{CC}$, or solely $1 \%$ FBS-HDMEM for $72 \mathrm{~h}$, before being subjected to TEM or fluorescence microscopy (magnification, x200). (C) Western blots showing protein levels of LC3 and beclin 1 in VSMCs treated with vehicle or $\beta$-GP. (D) Western blots showing protein levels of LC3 and beclin 1 in VSMCs treated with $\beta$-GP, metformin, or 3-MA. Western bands of interest were normalized against GAPDH, and data are presented as relative density ratios compared with control group or $\beta$-GP group. Representative images are shown. Data are presented as mean $\pm \mathrm{SEM}, \mathrm{n}=3$. ${ }^{*} \mathrm{P}<0.05,{ }^{* *} \mathrm{P}<0.01$. $\beta$-GP, $\beta$-glycerophosphate; Met, metformin; 3-MA, 3-methyladenine; CC, compound C; VSMC, vascular smooth muscle cell; TEM, transmission electron microscopy; LC3, microtubule-associated protein 1 light chain 3; HDMEM, high-glucose Dulbecco's modified Eagle's medium.

metformin could reduce calcification in the VSMCs induced by $\beta$-GP, the cells were first cultured in $1 \%$ FBS-HDMEM containing $\beta$-GP $(10 \mathrm{mmol} / \mathrm{l})$, and were then treated with metformin $(500 \mu \mathrm{mol} / \mathrm{l})$. When compared with the cells that were treated solely with $\beta$-GP, the calcium deposition was found to be reduced in the metformin-treated cells (Fig. 1C). Western blot analysis also revealed that the protein expression level of $\alpha$-SMA was increased, whereas the protein expression level of RUNX2 was decreased in metformin treated cells compared with the $\beta$-GP only treated cells (Fig. 1D). These results suggest that metformin could potentially alleviate $\beta$-GP-induced calcification of VSMCs.

Metformin promotes $\beta$-glycerophosphate-induced autophagy in VSMCs. To elucidate the potential effect of metformin on autophagy in VSMCs, the cells were subjected to TEM to examine their autophagic activity. TEM results revealed an increase in the accumulation of autophagosomes in the cells that were treated with $\beta$-GP compared with untreated control cells, which was found to be further increased in cells that were treated with both $\beta$-GP and metformin (Fig. 2A). Fluorescence microscopy results showed that the formation of the green fluorescent LC3 puncta in VSMCs increased after treatment with $\beta$-GP when compared to the cells that were treated only with the vehicle, and the green fluorescent LC3 puncta were found to be further increased after treatment with both $\beta$-GP and metformin (Fig. 2B). Additionally, the protein expression levels of LC3II/I and beclin 1 were examined in the VSMCs using western blot analysis. As shown in Fig. 2C, the expression levels of LC3II/I and beclin 1 were significantly increased in VSMCs that were treated with $\beta$-GP as compared with cells treated with vehicle. After finding that $\beta$-GP increased the protein expression levels of LC3II/I and beclin 1 compared to the untreated control group, further experiments compared cells treated with multiple drugs with $\beta$-GP alone. $\beta$-GP and metformin treatment further increased the expression levels of LC3II/I and beclin 1 in comparison to $\beta$-GP alone (Fig. 2D). These results suggest that $\beta$-GP may potentially stimulate autophagy in VSMCs and that metformin may further promote autophagic activity. 

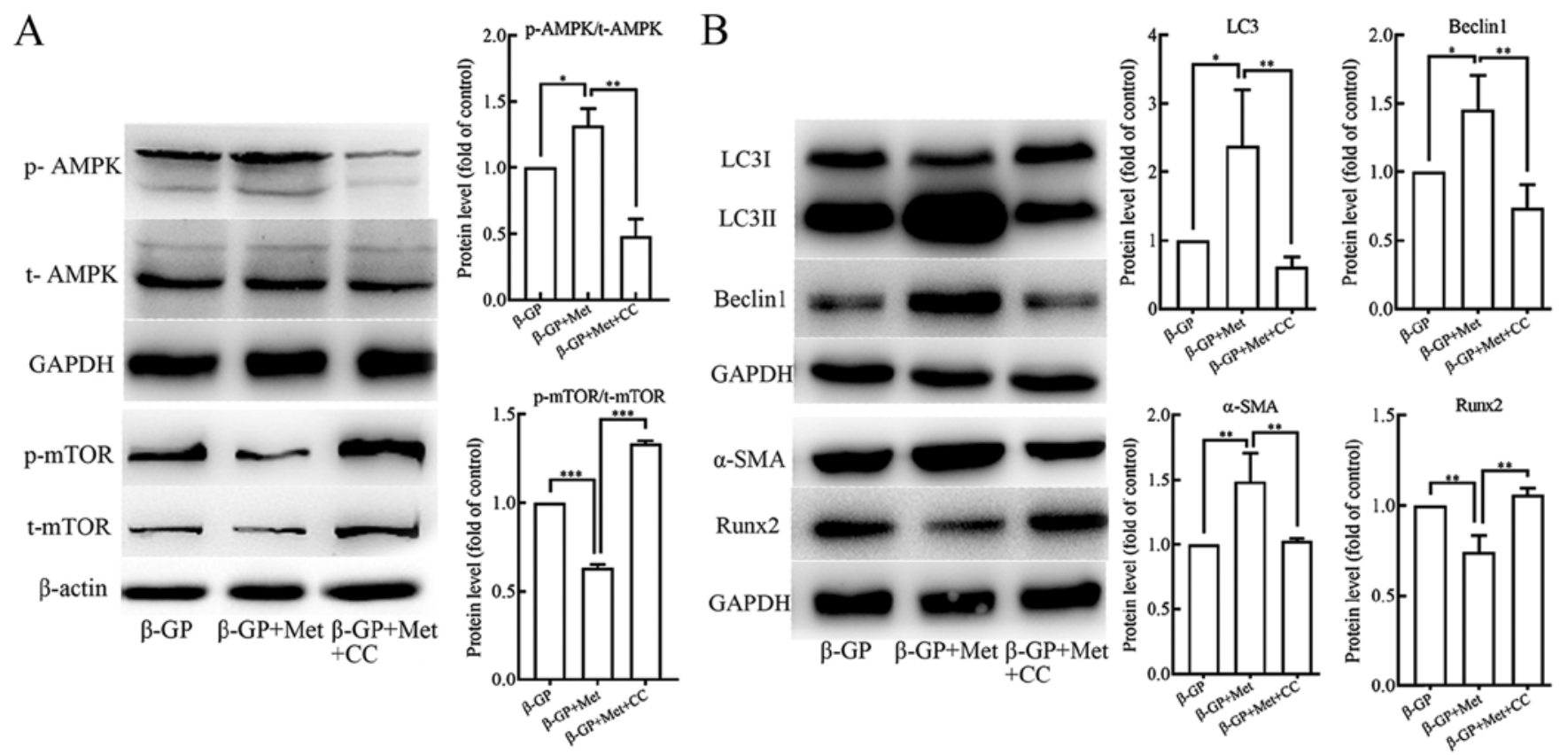

Figure 3. The effects of $\beta$-GP, Met and CC on the AMPK signaling pathway and on cell calcification and autophagy activity in VSMCs. (A) Western blots showing protein levels of $\mathrm{p}$ - and t-AMPK and mTOR in VSMCs treated with $\beta$-GP, Met or CC. (B) Western blots showing protein levels of LC3, beclin 1, $\alpha$-SMA and Runx 2 in VSMCs treated with $\beta$-GP, MET, or CC. Western bands of interest were normalized against GAPDH or $\beta$-actin, and data are presented as relative density ratios compared with $\beta$-GP group. Representative images are shown. Data are presented as mean $\pm \mathrm{SEM}, \mathrm{n}=3$. ${ }^{*} \mathrm{P}<0.05,{ }^{* *} \mathrm{P}<0.01,{ }^{* * * *} \mathrm{P}<0.001$. $\beta$-GP, $\beta$-glycerophosphate; Met, metformin; 3-MA, 3-methyladenine; CC, compound C; VSMC, vascular smooth muscle cell; p, phosphorylated; t, total; LC3, microtubule-associated protein 1 light chain 3; $\alpha$-SMA, $\alpha$-smooth muscle actin; RUNX2, runt-related transcription factor 2; AMPK, 5' adenosine monophosphate-activated protein kinase; mTOR, mammalian target of rapamycin.

Metformin alleviates $\beta$-glycerophosphate-induced VSMC calcification by enhancing autophagy. To further determine whether metformin alleviated the $\beta$-GP-induced VSMC calcification by promoting autophagy in VSMCs, cells were pre-treated with the autophagy inhibitor, 3-MA $(5 \mathrm{mmol} / \mathrm{l})$ for $30 \mathrm{~min}$ before treatment with $\beta$-GP and metformin. The results of Alizarin Red S staining and an O-cresolphthalein complexone assay (Fig. 1C) showed an evident increase in calcium deposition in VSMCs that were pre-treated with 3-MA when compared to the cells that were not subjected to 3-MA pre-treatment. However, the number of autophagosomes observed under TEM (Fig. 2A) and green fluorescent LC3 puncta observed under the fluorescence microscope (Fig. 2B) were significantly decreased. Western blot analyses revealed significantly lower levels of LC3II/I and Beclin 1 in VSMCs that were pre-treated with 3-MA when compared to the cells that were not subjected to 3-MA pre-treatment (Fig. 2D). In addition, the protein expression level of $\alpha$-SMA was found to be reduced, whereas the protein expression level Runx 2 was found to be increased when compared to the cells that were not subjected to 3-MA pre-treatment (Fig. 1D). Therefore, these results indicate that metformin alleviates $\beta$-GP-induced VSMC calcification by promoting autophagic activity.

Metformin promotes autophagy by activating the AMPK/mTOR signaling pathway. In order to understand the role of the AMPK/mTOR signaling pathway in the induction of autophagy by metformin, VSMCs were pre-treated with AMPK inhibitor compound $\mathrm{C}(10 \mu \mathrm{mol} / \mathrm{l})$ for $30 \mathrm{~min}$ before treatment with $\beta$-GP and metformin. Western blot analyses revealed that p-AMPK levels were increased and p-mTOR levels were reduced in
VSMCs that were treated with both, $\beta$-GP and metformin when compared to the cells that were treated only with $\beta$-GP (Fig. 3A). In addition, the protein expression levels of LC3II/I and Beclin 1 were shown to be significantly increased (Fig. 3B), and also the protein expression level of $\alpha$-SMA was increased, but the protein expression levels of Runx 2 was found to be decreased (Fig. 3B) in the cells that were treated with both $\beta$-GP and metformin in comparison to the cells that were treated only with $\beta$-GP. When the VSMCs were pre-treated with the Compound $\mathrm{C}$, a significant reduction in p-AMPK and increase in p-mTOR levels was observed (Fig. 3A). In addition, the protein expression levels of LC3II/I and Beclin 1 were found to be reduced (Fig. 3B) along with the expression level of $\alpha$-SMA, but the protein expression level of Runx2 was found to be increased (Fig. 3B). The number of autophagosomes observed under TEM (Fig. 2A) and the green fluorescent LC3 puncta observed under the fluorescence microscope (Fig. 2B) were evidently reduced in the VSMCs that were pre-treated with compound C. However, the results of Alizarin Red S staining and and an O-cresolphthalein complexone assay (Fig. 1C) showed a significant increase in the levels of calcium deposition. These results suggested that metformin could activate the AMPK/mTOR signaling pathway in VSMCs that were treated with $\beta$-GP, leading to an enhancement in cellular autophagic activity, in turn alleviating the $\beta$-GP-induced calcification of the VSMCs.

\section{Discussion}

The occurrence of vascular calcification in patients with CKD is thought to be due to the transformation of vascular smooth muscle cells from a contractile to an osteogenic 
phenotype, which may be induced by hyperphosphatemia (18). However, the mechanisms underlying this transformation remain to be elucidated. In the present study, using an in vitro cellular model of VSMC calcification, it was demonstrated that metformin inhibited the VSMC contractile to osteogenic phenotypic transformation induced by $\beta$-GP and thus reduced their calcification. In addition, the results suggested that metformin alleviated the calcification of VSMCs by promoting their autophagic activity, which was indicated by an increased number of autophagosomes, green fluorescent LC3 puncta and expression levels of LC3II/I and Beclin 1 in metformin treated cells when compared with controls. These metformin-induced increases were later reversed by treatment with the autophagy inhibitor 3-MA. Metformin appeared to promote the autophagic activity of VSMCs by activating the AMPK/mTOR signaling pathway, as indicated by the elevated levels of p-AMPK and reduced levels of p-mTOR that were thought to be induced by metformin when compared with a control and could be reversed by treatment with compound $\mathrm{C}$.

Metformin is widely used in the treatment of type 2 diabetes in clinical practice, typically at an oral dose of $500-1,000 \mathrm{mg}$ in the form of standard immediate-tablet formulation (19). Metformin has also been shown to exhibit protective effects on the cardiovascular system. Previous clinical studies demonstrated that metformin could markedly improve below-the-knee arterial calcification in patients with type 2 diabetes (20) and coronary atherosclerosis in a diabetes prevention study (21). Additionally, in an in vivo study in rats, it was demonstrated that metformin could potentially alleviate vitamin D3- and nicotine-induced vascular calcification via the AMPK pathway (22). In cellular biology experiments, metformin has also been shown to inhibit nuclear factor- $\kappa \mathrm{B}(23)$ and activate the AMPK-phosphatase and tensin homolog pathways (24) to alleviate the inflammatory response in human and rat VSMCs, respectively. Furthermore, a study indicated that metformin inhibited human aortic VSMC proliferation and migration through activation of the AMPK signaling pathway and up-regulation of expression levels of p53 and interferon $\gamma$-inducible protein 16 (25). In the present study, it was demonstrated that metformin increased the expression level of $\alpha$-SMA and down-regulated the expression level of Runx2 when compared with a control treatment. Additionally, it was demonstrated that metformin could significantly alleviate calcium deposition in VSMCs in in vitro experiments. Taken together these results suggested that metformin potentially repressed $\beta$-GP-induced contractile-to-osteogenic phenotypic switching in VSMCs and alleviated their calcification.

Autophagy is involved in the degradation of damaged and senescent organelles, as well as the long lived proteins, which is critical for survival, development and differentiation of cells, and also for the maintenance of homeostasis (26). It is a conserved cellular process that takes place in all the eukaryotic cells and plays a pivotal role in multiple human diseases (27). It has previously been demonstrated that autophagy dysregulation is involved in a wide array of vascular diseases, and also in the arterial medial calcification of the tunica media in chronic kidney diseases (8). In a study that was performed using a diabetes mouse model, metformin was found to prevent cardiomyopathy by up-regulating the autophagic activity in the myocardial cells (28). The present study indicated that
$\beta$-GP increased LC3II/I expression in comparison to the control group, suggesting that cellular autophagic activity was activated in a high phosphate environment. This is supported by the findings of previous publications that demonstrated that $\beta$-GP could promote cellular autophagic activity $(9,29)$. In the present study, metformin could further stimulate the autophagic activity in the VSMCs treated with $\beta$-GP, as shown by the marked increase in the number of autophagosomes, green fluorescent LC3 puncta and LC3II/I and Beclin 1 protein expression levels in comparison with controls. Intracellular accumulation of the LC3 puncta observed under fluorescence microscope has been shown to be associated with autophagic activity in cells (30). In the present study, when the cells were pre-treated with the frequently used autophagy inhibitor, 3-MA, before treatment with both $\beta$-GP and metformin (31) an increase in the number of autophagosomes and green fluorescent LC3 puncta and a reduction the expression levels of LC3II/I and Beclin 1 were observed in comparison to cells that had not been pre-treated. In addition, the $\alpha$-SMA expression level was decreased, but the expression level of Runx 2 and calcium deposition was found to be increased after treatment with 3-MA in comparison with untreated cells. These results suggest that the effect of metformin on the phenotypic switching and subsequent calcification of the VSMCs may be due to stimulation of the autophagic activity in the VSMCs.

The AMPK/mTOR signaling is an important pathway involved in the process of cellular autophagy. AMPK directly phosphorylates unc-51-like autophagy activating kinase 1 (ULK1) and subsequently activates autophagy (32), however, it may also deactivate the mTORC1 complex, which prevents the phosphorylation of ULK1 and its association with AMPK, and thus may indirectly activate the process of autophagy (12). By investigating the mechanism by which metformin activated the autophagic activity in the VSMCs, it was demonstrated that metformin increased the phosphorylation of AMPK and reduced phosphorylation of mTOR in VSMCs that were treated with $\beta$-GP. In addition, the protein expression levels of the autophagy-associated proteins, including LC3II/I and Beclin 1, as well as the number of autophagosomes and green fluorescent LC3 puncta were found to be increased. Moreover, the protein expression level of $\alpha$-SMA was also increased, but the protein expression level of Runx 2 and calcium content were decreased. However, upon treatment of these cells with the AMPK inhibitor, Compound C, the expression levels of p-AMPK and p-mTOR were markedly reversed, and the expression levels of the autophagy-associated proteins and $\alpha$-SMA were decreased, but the expression level of Runx 2 and calcium deposition were increased. Therefore, these results indicate that the effect of metformin on the autophagic activity of the VSMCs was implemented, at least in part, by activation of the AMPK/mTOR signaling pathway, which subsequently inhibited the phenotypic transition towards osteogenesis in high-phosphorus milieu and thus alleviated its calcification.

The major causes of lethality in patients with CKD are the cardiovascular events (33). As vascular calcification is an independent risk factor contributing to the lethal cardiovascular events, it is necessary to decelerate the progression of vascular calcification in these patients and to implement it in the clinical practices. In a study conducted using a rat model of CKD, which was induced by an adenine diet, metformin 
was shown to protect the rats from developing severe CKD by reducing the inflammation and fibrosis in the kidney, as well as exhibited a favorable effect on the associated comorbidities such as vascular calcification (34).

Several limitations must be contemplated while considering the results of the present study. In the present study, VSMCs were treated with $\beta$-glycerophosphate, metformin, 3-methyladenine, and/or compound $\mathrm{C}$ to induce calcification, to examine the effect of metformin on calcification and to investigate the role of autophagy, along with the involvement of the AMPK/mTOR signaling pathway. However, this is a preliminary study that, was conducted using a model system and to address the hypothesis. Therefore, future studies are warranted to further elucidate the underlying mechanisms. In addition, based on the fact that the living organisms respond to the pharmaceutical agents in a more complex manner, further in vivo studies based on animal models are necessary to before concluding the full biological effect of metformin on vascular calcification in a living organism. Moreover, even if it seems to be a very promising and novel interventional method for the treatment of vascular calcification in patients with a chronic kidney disease, a number of pre-clinical and clinical studies should be performed before the direct implementation of metformin in clinical practices.

In the present study, it was demonstrated that metformin could promote cellular autophagy and could potentially exert a beneficial impact on the $\beta$-GP-induced calcification of the VSMCs by activating the AMPK/mTOR signaling pathway. Therefore, metformin may exhibit a potential role in reducing vascular calcification independent of its hypoglycemic action. Nevertheless, as metformin may also induce lactate acidosis in CKD patients, its use in these cohorts is limited (35). Lalau et al (36) have previously reported that in moderate-to-severe CKD patients, it is possible to adjust the dose of metformin according to patient renal function, in order to exert a safe but a pharmacologically efficacious function. In general, apart from reducing the blood glucose levels, metformin may exhibit a dual function in protecting the kidney, as well as in alleviating the vascular calcification (34). In addition, in the work conducted by Zhang et al (22), the role of metformin in vitamin D3 and nicotine-induced vascular calcification was investigated. In this study, a dose of $300 \mathrm{mg} / \mathrm{kg}$ metformin was administered to 170-200 g rats, which was clearly an overdose for the CKD patients with a compromised renal function. In the present study, $500 \mu \mathrm{mol} / 1$ metformin was used in the treatment of VSMCs, which was based on an established protocol for in vitro experiments (15). In the future, it will be important to conduct in vivo experiments in order to further investigate the role of metformin, potentially by using a rat model of vascular calcification with compromised renal function. Also, the dose of metformin should be carefully tested and adjusted in order to provide a potential therapeutic value to its clinical usage in CKD patients.

In the present study, it was demonstrated that metformin, apart from its most common use in reducing blood glucose levels in diabetic patients, could also exert a beneficial effect on the $\beta$-GP-induced calcification of the VSMCs by promoting the cellular autophagic activity and activating the
AMPK/mTOR signaling pathway. Therefore, we could provide relevant information regarding a novel therapeutic agent that could be applied in the treatment of vascular calcification in CKD patients; however, further studies should be performed in the future to better understand the underlying mechanisms and validate the effectiveness of metformin.

\section{Acknowledgements}

The authors would like to thank Dr Zhiyuan Wu [University of ErlangenNuremberg, Germany (Friedrich-Alexander-Universität Erlangen-Nürnberg)] for assistance with imaging.

\section{Funding}

This work was supported by grants from the National Natural Science Foundation of China (grant no. 81770766) and the Provincial Natural Science Foundation of Liaoning (grant no. 20170540999). Professor Li Yao was also funded by the Shenyang Scientific Innovation Research and Development Project (grant no. 1900192) and Dr Tianhua Xu was also funded by Youth Mainstay Supporting Project of China Medical University (grant no. QGZD2018005).

\section{Availability of data and materials}

The datasets used and/or analyzed during the current study are available from the corresponding author on reasonable request.

\section{Authors' contributions}

XQ and LY designed the experiments; XQ performed the majority of experiments; QX performed the immunofluorescence experiment; TX and PW conducted the TEM; ZS and YH performed all the statistical analysis; XQ, QX, TX and LY wrote the manuscript. All the authors read and approved the final manuscript.

\section{Ethics approval and consent to participate}

Not applicable.

\section{Patient consent for publication}

Not applicable.

\section{Competing interests}

The authors declare that they have no competing interests.

\section{References}

1. Coresh J: Update on the burden of CKD. J Am Soc Nephrol 28: 1020-1022, 2017.

2. Go AS, Chertow GM, Fan D, McCulloch CE and Hsu CY: Chronic kidney disease and the risks of death, cardiovascular events, and hospitalization. N Engl J Med 351: 1296-1305, 2004.

3. Bardeesi ASA, Gao J, Zhang K, Yu S, Wei M, Liu P and Huang H: A novel role of cellular interactions in vascular calcification. J Transl Med 15: 95, 2017. 
4. Cannata-Andia JB and Martin KJ: The challenge of controlling phosphorus in chronic kidney disease. Nephrol Dial Transplant 31: 541-547, 2016.

5. Durham AL, Speer MY, Scatena M, Giachelli CM and Shanahan CM: Role of smooth muscle cells in vascular calcification: Implications in atherosclerosis and arterial stiffness. Cardiovasc Res 114: 590-600, 2018.

6. Tai S, Hu XQ, Peng DQ, Zhou SH and Zheng XL: The roles of autophagy in vascular smooth muscle cells. Int J Cardiol 211: 1-6, 2016.

7. Salabei JK and Hill BG: Autophagic regulation of smooth muscle cell biology. Redox Biol 4: 97-103, 2015.

8. Nussenzweig SC, Verma S and Finkel T: The role of autophagy in vascular biology. Circ Res 116: 480-488, 2015.

9. Dai XY, Zhao MM, Cai Y, Guan QC, Zhao Y, Guan Y, Kong W, Zhu WG, Xu MJ and Wang X: Phosphate-induced autophagy counteracts vascular calcification by reducing matrix vesicle release. Kidney Int 83: 1042-1051, 2013.

10. Piwkowska A, Rogacka D, Jankowski M, Dominiczak MH, Stepinski JK and Angielski S: Metformin induces suppression of $\mathrm{NAD}(\mathrm{P}) \mathrm{H}$ oxidase activity in podocytes. Biochem Biophys Res Commun 393: 268-273, 2010.

11. Kim J, Kundu M, Viollet B and Guan KL: AMPK and mTOR regulate autophagy through direct phosphorylation of Ulk1. Nat Cell Biol 13: 132-141, 2011.

12. Egan D, Kim J, Shaw RJ and Guan KL: The autophagy initiating kinase ULK1 is regulated via opposing phosphorylation by AMPK and mTOR. Autophagy 7: 643-644, 2011.

13. Wang S, Song P and Zou MH: AMP-activated protein kinase, stress responses and cardiovascular diseases. Clin Sci (Lond) 122: 555-573, 2012.

14. Shioi A, Nishizawa Y, Jono S, Koyama H, Hosoi M and Morii $\mathrm{H}$ Beta-glycerophosphate accelerates calcification in cultured bovine vascular smooth muscle cells. Arterioscler Thromb Vasc Biol 15: 2003-2009, 1995.

15. Cao X, Li H, Tao H, Wu N, Yu L, Zhang D, Lu X, Zhu J, Lu Z and Zhu Q: Metformin inhibits vascular calcification in female rat aortic smooth muscle cells via the AMPK-eNOS-NO pathway. Endocrinology 154: 3680-3689, 2013.

16. She C, Zhu LQ, Zhen YF, Wang XD and Dong QR: Activation of AMPK protects against hydrogen peroxide-induced osteoblast apoptosis through autophagy induction and NADPH maintenance: New implications for osteonecrosis treatment? Cell Signal 26: 1-8, 2014

17. Zhu D, Mackenzie NC, Shanahan CM, Shroff RC, Farquharson C and MacRae VE: BMP-9 regulates the osteoblastic differentiation and calcification of vascular smooth muscle cells through an ALK1 mediated pathway. J Cell Mol Med 19: 165-174, 2015.

18. Shroff R, Long DA and Shanahan C: Mechanistic insights into vascular calcification in CKD. J Am Soc Nephrol 24: 179-189, 2013.

19. Bailey CJ: Metformin: Historical overview. Diabetologia 60: 1566-1576, 2017.

20. Mary A, Hartemann A, Liabeuf S, Aubert CE, Kemel S, Salem JE, Cluzel P, Lenglet A, Massy ZA, Lalau JD, et al: Association between metformin use and below-the-knee arterial calcification score in type 2 diabetic patients. Cardiovasc Diabetol 16: 24, 2017

21. Goldberg RB, Aroda VR, Bluemke DA, Barrett-Connor E, Budoff M, Crandall JP, Dabelea D, Horton ES, Mather KJ, Orchard TJ, et al: Effect of long-term metformin and lifestyle in the diabetes prevention program and its outcome study on coronary artery calcium. Circulation 136: 52-64, 2017.
22. Zhang X, Xiao J, Li R, Qin X, Wang F, Mao Y, Liang W, Sheng X, Guo M, Song Y and Ji X: Metformin alleviates vascular calcification induced by vitamin D3 plus nicotine in rats via the AMPK pathway. Vascular pharmacol 81: 83-90, 2016.

23. Isoda K, Young JL, Zirlik A, MacFarlane LA, Tsuboi N, Gerdes N, Gerdes N, Schönbeck U and Libby P: Metformin inhibits proinflammatory responses and nuclear factor-kappaB in human vascular wall cells. Arterioscler Thromb Vasc Biol 26: 611-617, 2006.

24. Kim SA and Choi HC: Metformin inhibits inflammatory response via AMPK-PTEN pathway in vascular smooth muscle cells. Biochem Biophys Res Commun 425: 866-872, 2012.

25. Hao B, Xiao Y, Song F, Long X, Huang J, Tian M, Deng S and Wu Q: Metformin-induced activation of AMPK inhibits the proliferation and migration of human aortic smooth muscle cells through upregulation of p53 and IFI16. Int J Mol Med 41: 1365-1376, 2018.

26. Feng Y, He D, Yao Z and Klionsky DJ: The machinery of macroautophagy. Cell Res 24: 24-41, 2014.

27. Choi AM, Ryter SW and Levine B: Autophagy in human health and disease. N Engl J Med 368: 651-662, 2013.

28. Xie Z, Lau K, Eby B, Lozano P, He C, Pennington B, Li H, Rathi S, Dong Y, Tian R, et al: Improvement of cardiac functions by chronic metformin treatment is associated with enhanced cardiac autophagy in diabetic OVE26 mice. Diabetes 60: 1770-1778, 2011.

29. Xu M, Liu L, Song C, Chen W and Gui S: Ghrelin improves vascular autophagy in rats with vascular calcification. Life Sci 179: 23-29, 2017.

30. Klionsky DJ, Abdelmohsen K, Abe A, Abedin MJ, Abeliovich H, Acevedo Arozena A, Adachi H, Adams CM, Adams PD, Adeli $\mathrm{K}$, et al: Guidelines for the use and interpretation of assays for monitoring autophagy (3rd edition). Autophagy 12: 1-222, 2016.

31. Mizushima N, Yoshimori T and Levine B: Methods in mammalian autophagy research. Cell 140: 313-326, 2010.

32. Egan DF, Shackelford DB, Mihaylova MM, Gelino S, Kohnz RA, Mair W, Vasquez DS, Joshi A, Gwinn DM, Taylor R, et al: Phosphorylation of ULK1 (hATG1) by AMP-activated protein kinase connects energy sensing to mitophagy. Science 331: 456-461, 2011.

33. Webster AC, Nagler EV, Morton RL and Masson P: Chronic kidney disease. Lancet 389: 1238-1252, 2017.

34. Neven E, Vervaet B, Brand K, Gottwald-Hostalek U, Opdebeeck B, De Mare A, Verhulst A, Lalau JD, Kamel S, De Broe ME and D'Haese PC: Metformin prevents the development of severe chronic kidney disease and its associated mineral and bone disorder. Kidney Int 94: 102-113, 2018.

35. Lipska KJ, Bailey CJ and Inzucchi SE: Use of metformin in the setting of mild-to-moderate renal insufficiency. Diabetes Care 34: 1431-1437, 2011.

36. Lalau JD, Kajbaf F, Bennis Y, Hurtel-Lemaire AS, Belpaire F and De Broe ME: Metformin treatment in patients with type 2 diabetes and chronic kidney disease stages $3 \mathrm{~A}, 3 \mathrm{~B}$, or 4 . Diabetes Care 41: 547-553, 2018.

(i) $($ This work is licensed under a Creative Commons Attribution-NonCommercial-NoDerivatives 4.0 International (CC BY-NC-ND 4.0) License. 